\title{
Beiträge zur Geschichte der Erkenntnis des Erdmagnetismus
}

\author{
Von Heinz BaLmer, Konolfingen (Bern)
}

Die Mittelmeervölker des Altertums kannten die magnetische Richtungsweisung nicht. Doch lief die Kunde um, daß der Magnetstein, den man in Indien, Äthiopien, an den Gestaden der Ägäis und in Spanien fand, Eisen anziehe. Lukrez vermutete ausströmende Magnetatome, in deren Feld das Eisen, von hinten durch den Luftdruck getrieben, vorwärtsstürze. Ärzte hofften, daß der Stein auch Krankheiten ansauge, und Seeleute fürchteten, daß magnetische Felsen von ferne die Schiffsnägel anziehen könnten. Wenn der Stein seine Kraft verlor, sollte der Geruch des Knoblauchs oder die Nähe eines Diamanten ihn besiegt haben.

Die Nachricht, daß ein vom Magneten angeregtes Eisenstäbchen nach Norden weise, tauchte um 1200 in Frankreich auf. Man steckte das Stäbchen quer durch einen Strohhalm, der auf dem Wasser lag, oder durch eine senkrechte Achse, die sich zwischen Boden und Glasdeckel einer Schachtel drehte. Im 13. Jahrhundert breitete sich das Gerät in Arabien, Spanien und Italien aus. Die Nadel schien sich nach dem Polarstern oder nach einer Magneterzgrube im hohen Norden zu richten.

1269 schrieb der französische Astronom und Optiker Petrus PeregriNUS DE MARICOURT aus Unteritalien einen Brief über den Magneten, worin er mit einer Klarheit, die 300 Jahre unerreicht blieb, eine Versuchskette darlegt. Die Schrift trägt den Stempel seiner schöpferischen Persönlichkeit. Er war als Instrumentenbauer im Messen von Gestirnsazimuten und im Schleifen von Linsen geübt. Den Magnetstein kannte er von den Seeleuten der heimatlichen Picardie.

Er sah, daß der Stein, wenn man ihn in einer Holzschale aufs Wasser legte, sich wie eine Eisennadel nach Norden und Süden einstellte. Um eine in $360 \mathrm{Grad}$ eingeteilte Scheibe zur Messung der Gestirnsazimute polwärts zu richten, nahm er einen Stein, schliff ihn wie ein Glas in Kugelform, fügte ihn in die Schachtel unter der Scheibe und legte das Ganze aufs Wasser. Er meinte, der Stein richte sich weder nach einer Magneterzgrube noch nach dem Polarstern, sondern genau nach den beiden Himmelspolen. Eine drehbare Leiste auf der Scheibe diente zur Messung der Meridianabweichungen der Sonne oder der Sterne und damit zur Zeitbestimmung. Peregrinus erblickte im runden Magnetstein ein kleines Himmelsmodell, dessen 
zwei Seiten er nach Übereinstimmung mit dem Himmel «Pole» benannte, Nur die tägliche Drehung, die man dem Himmelsgewölbe beilegte, fehlte beim Abbild. Doch nahm der Forscher an, daß ein vollkommen runder Stein bei richtiger Achsenneigung sich ewig täglich um die Achse drehen sollte. Er untersuchte die Anziehung und Abstoßung zwischen den Polen zweier Steine. Jedes Magnetbruchstück zeigte wieder zwei Pole. Ein Eisennädelchen richtete sich auf der Oberfläche des Steins nach dessen Polen. Das mit dem Südpol des Magneten berührte Nadelende kehrte sich hernach auf dem Wasser nordwärts. Peregrinus kannte außer dem Schwimmkompaß auch die Nadel, die sich samt der senkrechten Achse zwischen Boden und Glasdeckel einer Schachtel drehte. Auch hier teilte er den Deckel in $360 \mathrm{Grad}$ und maß mit einer Leiste Gestirnswinkel. Endlich schwebte ihm vor, der Plan eines ewig beweglichen Rädchens lasse sich verwirklichen, indem man es ringsum mit Nägeln beschlage und einen Magneten in die Nähe setze.

Nicht die klaren Versuche, wohl aber die Hoffnungen auf das von selber umlaufende Kügelchen oder Rädchen sicherten dem Briefe Leser bis ins 16. Jahrhundert. Damals mußten sich die Anhänger des Peregrinus mit zwei neuen Erkenntnissen auseinandersetzen: daß nicht der Himmel, sondern die Erde sich um Pole drehe, und daß die Magnete nicht genau polwärts zeigten. -

In der Hafenstadt Amalfi im Königreich Neapel soll um 1300 der Kompaß die für die Seefahrt geeignete Form gewonnen haben. Hatte sich bisher die Nadel samt der Achse unter dem festen Teilkreis bewegt und durch ihre Schwankungen die Ablesung erschwert, so wurde nun der Teilkreis in Form einer Papierscheibe mit der Nadel drehbar verbunden und auf die Spitze einer unbeweglichen Achse gesetzt. Die Schiffskompasse wurden bald auch in Genua, Spanien und Flandern hergestellt.

Der Teilkreis vereinfachte sich zur Windrose. Sie hatte 32 Windstriche (spanisch rumbos) in Abständen von je 11/1/4 Grad, eine Lilie im Norden und zuweilen ein Kreuzchen im Osten. Die acht Hauptwinde waren schwarz, die acht halben Winde grün und die sechzehn Viertelwinde rot gezogen, ebenso auf den Seekarten. An die Unterseite der Kartonscheibe klebte man das magnetisierte Eisenstück. Die damaligen Nadeln hatten meist Drahtform (englisch the wiers, lateinisch ferramenta). Daher pflegte man das Hütchen in der Mitte der Kartonscheibe anzubringen und das zu magneti, sierende Drähtchen so zu einem Bügel oder Spindelumriß zu krümmen, daß seine beiden Schenkel links und rechts neben dem Hütchen vorbei- 
liefen. Oder man nahm zwei Drähte, deren Enden sich trafen. Das ganze Drehgerät schwebte auf einem Stift im Innern einer glasbedeckten runden Holzschachtel. Diese wurde im 16. Jahrhundert mit einem Bleigewicht beschwert und hing an einer waagrechten Achse innerhalb eines waagrechten Ringes, der seinerseits an einer Querachse innerhalb eines zweiten Ringes hing. Dank diesen kreuzweisen Achsen hielt die Büchse bei jedem Wellengang die Schwerpunktslage ein. Ein solcher Kompaß konnte manchen Fehler haben. Zuweilen lagen die Drahtenden nicht in gerader Linie mit dem Unterstützungspunkte. Sie konnten auch zuwenig magnetisiert oder verrostet sein. Oder die Windrose neigte sich auf einer Seite abwärts. Der Tragstift war verkrümmt oder abgestumpft. Zuweilen ließ die Schachtel Luftzug herein oder drehte sich schwer in den Angeln der cardanischen Aufhängung.

Mit den ersten Schiffskompassen las man im Mittelmeer die Windrichtungen von Landspitze zu Landspitze ab und zeichnete die Küsten. Die Teilaufnahmen wurden zu Mittelmeerkarten vereinigt. Darauf erscheinen die nach dem Kompaß gezeichneten Küsten gegenüber den festen Himmelsrichtungen der Karte im Gegenuhrzeigersinne verschoben. Die magnetische Abweichung war also damals im Mittelmeer östlich. Im rechten Teil des Meeres ist die Verdrehung stärker.

Die Abweichung wurde erst näher beachtet, als Kolumbus auf seinen Fahrten durch den Atlantischen Ozean entdeckte, daß die Abweichung seiner flämischen und genuesischen Kompasse jenseits der Azoren von der östlichen in eine westliche überging. Damit erwies sich die Abweichung als wechselnde Größe; doch schien es vorerst, als ob der Wechsel nur im Zusammenhang mit den Meridianen erfolge. Die Linie ohne Abweichung, die, wie man annahm, vom Nordpol zum Südpol verlief, wurde als Demarkationslinie zwischen den portugiesischen Hoheitsgebieten diesseits und den spanischen jenseits festgesetzt. Dadurch wurde die Bestimmung der Abweichung den Königen wichtig. Der Vergleich der Nadelrichtung mit der Stellung des Polarsterns genügte nicht mehr. Ein Apotheker überreichte 1525 dem portugiesischen König ein Bestimmungsgerät für die wahre Nordsüdrichtung. Sie ergibt sich als die Richtung, in der die Sonne mittags den Scheitel ihrer Bahn erreicht und in der der kürzeste Schatten fällt. Da in zwei Zeitpunkten gleicher Sonnenhöhe vor- und nachmittags ein Stab gleich lange Schatten wirft, den einen mehr westlich, den andern mehr östlich, entspricht die Mittellinie dazwischen ebenfalls der Meridianrichtung, mit der man die Nadelrichtung zu vergleichen hat. Die spanischen und 
portugiesischen Lehrbücher der Schiffahrtskunde verbreiteten dieses Verfahren. Ein Brettchen mit dem schattenwerfenden Stifte wurde oberhalb der Windrose angebracht, oder man konnte das Hütchen der Windrose selbst zu einem Stift verlängern, oder man verwendete den schattenwerfenden Faden eines Lotes. Wenn man gleiche Sonnenhöhen messen wollte, errichtete man über der Bussole einen vertikalen Viertelkreis mit einem auf eine bestimmte Höhe stellbaren Zeiger, durch dessen Absehlöcher die Sonne vor- und nachmittags je einmal scheinen mußte, während man den Viertelkreis um die senkrechte Mittelachse drehte.

Auf dem Festlande wurde die Abweichung unabhängig von der Seefahrt entdeckt. Seit etwa 1462 stellte man in den mechanischen Werkstätten Nürnbergs tragbare Sonnenuhren her. Bevor sich daran die Zeit ablesen ließ, mußte man sie nach dem Meridian richten. Dazu versah man sie mit einer Magnetnadel. Das ganze Gerät hieß «Sonnenkompaß». Weil die östliche Abweichung im nördlichen Europa größer war als im Mittelmeer, wurde sie in Deutschland schon um 1500 wahrgenommen. In Nürnberg betrug sie ungefähr 10 Grad. Man deutete sie durch ein Strichlein unter der Magnetnadel an. Im Gegensatz zum Schiffskompaß war bei den Nadeln der Sonnenuhren das südliche Ende spitz. Das nördliche war T- oder Y-förmig geschmiedet.

Auch beim Landkartenzeichnen wurde der Kompaß gebraucht; denn wenn man von Türmen aus die Richtungen zu den umliegenden Ortschaften zu messen suchte, mußte vorerst der Teilkreis mit seinem Anfangspunkte nach Norden gerichtet werden.

Rheticus beschäftigte sich 1540 mit der Abweichung. Sie erschien ihm als Besonderheit vieler Nadeln, die sie wiederum von einzelnen Magnetsteinen erhalten hätten. Im Anschluß an Peregrinus glaubte er noch, es gebe Magnetsteine, die «den Strich Nord und Süd recht halten». Andere Steine aber besäßen eine Abweichung und übertrügen sie auf die Nadeln. Daher sei jeder Stein vorerst zu prüfen, indem man eine Nadel damit bestreiche und ihre Richtung mit der Mittagslinie vergleiche. Jede Nadel sollte die einmal erhaltene Richtung überall beibehalten. Den Danziger Schiffskompaßmachern empfahl er, den magnetischen Drahtbügel unter der Windrose so zu befestigen, daß sein Nordende um den Betrag der Abweichung von der Lilieversetzt sei. Dann zeige der Kompa $\beta$ « allenthalben recht». Die Ortsabhängigkeit der Abweichungswerte wurde von Rheticus verworfen, dagegen vom Sonnenuhrenkünstler Georg Hartmann 1544 angenommen.

Die schräge Anheftung der Drähte an der Windrose war wohl ein Brauch, der im nördlichen Europa schon lange bestand. Herstellungsorte des Schiffs- 
kompasses waren damals Sizilien, Genua und Venedig, Sevilla und Lissabon, La Rochelle, Bordeaux und Rouen, England, Flandern, Dänemark und Danzig. Während alle Mittelmeerkompasse das Nordende der Drähte genau unter dem Nordpunkt der Windrose trugen, war es an den Atlantischen Küsten, an der Nordsee und Ostsee üblich, die Drähte nach Nordosten abzudrehen. Die spanischen, portugiesischen, französischen und englischen Kompasse, die für die Fahrt nach Amerika dienten, hatten die Drähte um einen halben Windstrich versetzt. Die englischen für die Fahrt nach Nordrußland hatten sie um zwei Drittel, die flandrischen und dänischen für die Nordsee um drei Viertel eines Windstrichs verschoben, endlich die Danziger für die Ostsee sogar um einen ganzen Windstrich $\left(11^{1 / 4} \mathrm{Grad}\right)$.

Die Küsten des Mittelmeeres, der Nordsee, der Ostsee, des Eismeeres und Amerikas wurden je mit einer dieser fünf verschiedenen Kompaßformen aufgenommen. Mit einer englischen Bussole war daher die Seefahrt im Mittelmeer gefährlich. Man mußte für jede Karte den passenden Kompaß benützen. Die Karten der verschiedenen Meeresbecken durften nicht zusammengesetzt werden. Besonders unangenehm war, daß die Abweichung jenseits der Azoren westlich wurde. Denn was die verdrehten Kompasse diesseits an Richtigkeit gewannen, schlug ihnen jenseits zum Nachteil aus. Wenn man bei der Fahrt nach Amerika unterwegs die Drähte unter der Windrose verschob, stiftete dies nur Verwirrung. Ließ man sie in der alten Lage, so wurde die Westrichtung, die ohnehin durch die nun westliche Abweichung nach Süden gedrängt wurde, infolge der zusätzlichen Verdrehung noch mehr nach Süden gedreht. Indem man dem westlichen Strich der Windrose folgte, steuerte man in Wirklichkeit südwestwärts und langte auf einer zu niedrigen Breite an. Von der Südwestspitze Englands aus erreichte man so im Westkurs die Südspitze Neufundlands, die fast um 4. Grad südlicher liegt, wie die Polhöhenbestimmung zeigte. Damit die Seekarte dem Bedürfnis des nach dem Kompaß steuernden Seemannes entsprach, mußte aber dieser Endpunkt auf gleiche Höhe wie der Ausgangsort gezeichnet werden. Es blieb nichts übrig, als die Breitenskala Kanadas gegenüber der europäischen nach Norden hinaufzurücken. Die Küsten waren wie Kulissen gegeneinander verschoben.

Pedro de Medina ermaß, welche Folgen die wechselnde Richtung der Magnetnadel für die Schiffskurse und Seekarten haben müßte. Doch da er keine genauen Meßwerte kannte und keinen Grund anzugeben vermochte, warum die Nadeln abweichen sollten, zweifelte er am Vorhandensein der ganzen Erscheinung. 
Dagegen wies der Kartenzeichner Gerhard Mercator 1546 nach, daß die Abweichung sich wirklich in den kompaßtreuen Karten widerspiegle. Die Küsten waren nicht nur infolge der Mißweisung falsch nach den Himmelsgegenden ausgerichtet, sondern zudem infolge der örtlichen Verschiedenheit der Mißweisung verzerrt oder verbogen. Die Nordküste Afrikas bog sich ostwärts zu sehr nach Norden auf, und die Küste Kanadas zerrte sich nach Norden weit auseinander. Später entdeckte Mercator für diesen letzten Umstand noch einen andern Grund. Er lag in der Kartenprojektion. Da die Maschen des Gradnetzes auf der Erdkugel polwärts schmaler werden, auf der Karte aber nicht, so mußten notwendig, da man die schrägen Schiffskurse auch dort winkeltreu zeichnete, jene Maschen auch in ihrer Höhenrichtung künstlich erweitert werden. Mercator erhob dies zum Grundsatze der winkeltreuen, nach ihm benannten Projektion.

Die verdrehten Kompasse hatten die Abweichungsfehler nicht beseitigt. Schon der Eismeerfahrer William Borough klagte 1581, man hätte mit dieser Sitte lieber nie beginnen sollen. Doch nun hatte man bereits die Karten damit aufgenommen. Daher überlebte der unglückliche Brauch seine Zeit und versank erst allmählich, als 1634 erkannt wurde, daß die Abweichung sich nicht nur örtlich, sondern auch mit der Zeit veränderte.

Eine Erklärung, weshalb sich die Nadel richte, war schwierig zu finden. Da kein anziehender Magnet in der Nähe zu sein schien, suchte man einen Anziehungsort in der Ferne. Am Himmelsgewölbe war er kaum, da sich seine Lage in bezug auf die Nadel nicht verändern durfte. Eher kam eine noch unbekannte Stelle der Erdoberfläche im hohen Norden in Frage. Aus dem Norden, aus Norwegen, bezog man viele Magnetsteine; doch gab es auch in Deutschland und auf Elba Fundstätten. Der wahre Magnetpol als stärkster Anziehungspunkt schien irgendwo im Eismeer zu liegen. Der sagenhafte Magnetberg, den die Furcht sogleich dorthin verpflanzte, förderte diese Vorstellung.

Man ahnte ungeheure Fernwirkungen. Porta plante bereits eine magnetische Telegraphie. Zwei völlig gleich hergestellte Magnetnadeln sollten beide in gleicher Weise mit dem Alphabet umschrieben werden. Wenn man hernach die eine Nadel mit der Hand auf die verschiedenen Buchstaben einstelle, werde die zweite Nadel in der Ferne von selber die gleichen Bewegungen nachahmen.

Bei der Fahrt nach Westen schien sich die Abweichung von Ort zu Ort mit so großer Regelmäßigkeit zu verändern, daß der Gedanke nahelag, die Abweichung lasse sich als Mittel zur Längenbestimmung verwenden. Zu 
beiden Seiten des vorausgesetzten Meridians ohne Abweichung nahm die Mißweisung allmählich zu, und zwar diesseits die östliche und jenseits die westliche, als ob die Nadel stets nach einem Punkt hinwiese, der jenseits des Nordpols auf der Rückseite der Erde in der Verlängerung des Meridians ohne Abweichung läge. Dort müßten alle Richtungslinien der Magnetnadeln zusammentreffen.

Gerhard Mercator berechnete die Lage jenes irdischen Magnetpols voraus, indem er die Richtungen der Nadel von zwei Erdorten aus über den Globus hin bis zu ihrem Schnittpunkt im Norden Asiens verlängerte. Zuerst rechnete er von der Insel Walcheren und Danzig aus; später wiederholte er die Rechnung, indem er den Abweichungswert für Regensburg und den angenommenen Nullmeridian zugrunde legte. Da man von diesem nicht sicher war, ob er bei den Kapverdischen Inseln oder weiter westlich bei der Azoreninsel Corvo verlaufe, zog Mercator beide Möglichkeiten in Betracht und bestimmte auf jedem der entsprechenden Großkreise den Schnittpunkt. Auf seiner Weltkarte von 1569 sind beide gezeichnet. Der Pol, bezogen auf die Kapverden, war der wahrscheinlichere. Bisher hatte man die Längenzählung nur zwei Grad östlicher bei den Kanarischen Inseln begonnen. Mercator verlegte den Anfang der Zählung auf die Kapverden.

1581 suchte William Borough, 1599 Johannes Kepler ebenfalls jenen Pol zu berechnen. Borough ging von den Azoren und von London aus, Kepler von den Werten für Grönland und Nowaja Semlja, wonach er das Ergebnis mit dem Wert für Graz verglich und sicherte. Doch stimmten die Voraussagen nicht überein. Mercators drei Berechnungen hatten für die Entfernung des Magnetpols vom geographischen Nordpol 11 oder 16 $1 / 2$ oder 13 Grad ergeben; Borough erhielt dafür 25 Grad 44. Minuten, Kepler dagegen nur 61/2 Grad. Livio SANUTo nahm 1588 an, der nördliche Magnetpol sei 24 Grad vom Nordpol entfernt, und ein südlicher Magnetpol liege dem ersten auf der Erde gegenüber.

Indem man stets einen Großkreis ohne Abweichung voraussetzte, wurde die Erde in eine Hälfte mit östlicher und in eine Hälfte mit westlicher Abweichung zerschnitten. Doch die Seefahrten nach Nordrußland und nach Ostasien ergaben, daß die östliche Abweichung bereits beim Nordkap in Norwegen und bei der Südspitze Afrikas wieder in eine westliche hinüberwechselte. Erst bei Kanton in China und hinter Java wurde sie wieder östlich. Abermals suchte man die Ergebnisse in ein Schema zu pressen. Simon Stevin glaubte 1599, aus diesen Angaben schließen zu dürfen, daß im Raume von 160 Längengraden drei Meridiane ohne Abweichung bestün- 
den. 60 Grad umspanne das Feld östlicher Abweichung zwischen Corvo und Südafrika, 100 das Feld westlicher Abweichung zwischen Südafrika und China. Da er die Meridiane ohne Abweichung zu Großkreisen ergänzte, wurde die Erdoberfläche in sechs Schnitze oder «Parks» mit abwechselnd östlicher und westlicher Abweichung eingeteilt. Die Mittelnähte der gedachten Abschnitte sollten Meridiane der größten Abweichung sein. Solche Täuschungen waren möglich, weil die Längen zuwenig genau bestimmt werden konnten. Daher wußte man unter anderem nicht, daß das Nordkap weiter im Osten liegt als die Südspitze Afrikas.

Stevins Hoffnung aber war es gerade, durch die Abweichungen ließe sich die Längenbestimmung ersetzen. Daher forderte er die Seefahrer auf, Abweichungswerte zu sammeln. Der Engländer GILBERT anerkannte ein Jahr darauf den Gedanken, daß durch Abweichungstabellen die Lagebestimmung zur See und das Finden der Häfen erleichtert werde; doch mußte er die regelmäßige Verteilung der Werte ablehnen. Er zeigte, daß die Nadeln nicht überall auf dem Meridian von Corvo die gleiche Richtung einhalten, ebensowenig auf den andern als abweichungslos angenommenen Meridianen. Nautonnier, der Tafeln für die mutmaßlichen Abweichungswerte vieler Erdorte vorausberechnete, trat mit seinem Werke 1603 verspätet hervor. Die Nachmessungen widerlegten ihn bald.

In das 16. Jahrhundert fällt eine Entdeckung, die die weitere Erforschung des Erdmagnetismus wesentlich fördern sollte. Es ist die Entdeckung der magnetischen Neigung oder Inklination.

Bereits 1544, beobachtete Georg Hartmann in Nürnberg, daß eine Eisennadel, die zuerst im Gleichgewicht auf einer Spitze schwebte und erst hernach magnetisiert wurde, nun plötzlich ihre Nordspitze senkte. Er erkannte darin eine besondere Erscheinung, verfolgte sie indessen nicht weiter. Unabhängig von ihm erlebte später der Instrumentenmacher Robert Norman in London genau dasselbe. Gewöhnlich heftete er einfach ein Stückchen Wachs auf die Südseite der Nadel, bis sie wieder ins Gleichgewicht kam. Einst aber, als eine Nadel von bestimmter Länge bei ihm bestellt war, ließ er sich dazu verleiten, von der Nordseite ein Stück abzuschneiden. Im Ärger über die verpfuschte Arbeit besprach er seine wiederholte Beobachtung mit Freunden. Man hielt ihm vor, daß der Magnetismus ein schwerer Stoff zu sein scheine. Warum aber sollte dann stets das Nordende sinken, auch wenn man mit dem Magneten die Südseite der Nadel berührte? Einige Drahtstücke, die Norman zuerst in unmagnetischem, dann in magnetischem Zustande wog, hatten ihr Gewicht nicht verändert. 
Indem Norman den Gegenstand weiter untersuchte, wurde er der bedeutendste experimentelle Erforscher des Erdmagnetismus seit Peregrinus. Er dachte ebensosehr an den Fortschritt der Wissenschaft wie an die Ehre Gottes. Sein Scharfsinn und seine Belesenheit erlaubten ihm, aus den Beobachtungen Schlüsse zu ziehen.

Die Nadel pflegte sich auf dem senkrechten Stifte zu neigen, bis ihr Hütchen am Stifte anstieß und eine weitere Senkung verhinderte. Um zu prüfen, ob die Nadel sich um volle $90 \mathrm{Grad}$ neigen würde, wenn sie sich ungehemmt nach unten drehen könnte, stellte Norman eine besondere Nadel her, durch deren Mitte eine waagrechte Achse gesteckt war, die sich in zwei gegenüberliegenden Lagern drehte. Die Zurichtung war gleich, wie wenn man mittelalterliche Kompaßschachteln in die Vertikalebene umgelegt hätte. Die Nadel mit der waagrechten Achse stellte das erste Neigungsgerät dar. Die zunächst unmagnetische Nadel mußte genau im Gleichgewicht schweben. Sobald man sie mit dem Magneten berührte, sank ihr Nordende (wenn man es in die Richtung der Abweichung hielt) in London um 71 Grad 50 Minuten unter den Horizont und blieb in dieser Schräge stehen. Es war, als ob eine Fernkraft aus einem Punkt in der Tiefe der Erde das Nordende der Nadel niederzöge, oder als ob eine Anziehung von der Höhe des Himmels ihr Südende emporhöbe. Norman prüfte, ob sich die Erscheinung auch bei einem Draht im Wasser zeige. Er steckte den zunächst unmagnetischen Draht durch ein Korkkügelchen, so daß dieses Schwimmgürtelchen seine Mitte umgab. Dann verkleinerte er das Kügelchen, bis das Drähtchen ein wenig untersank und waagrecht unter dem Wasserspiegel im Innern der Flüssigkeit schwebte. Sobald er es magnetisierte, drehte es sich in die Richtung der Abweichung und zugleich in dieselbe Schräglage wie die Nadel des Neigungsgerätes. Wäre der Draht nun in seiner Schräge nach dem Grunde des Bechers gesunken oder aber zur Wasseroberfläche aufgestiegen, so hätte man gewußt, ob eine Anziehung von unten oder von oben einwirke. Da aber weder das eine noch das andere geschah, schloß Norman, es bestehe keine äußere Anziehungskraft, sondern nur ein inneres Streben des magnetischen Körpers, auf einen bestimmten Zielpunkt im Erdinnern hinzudeuten. Er sah voraus, daß die Nadel an andern Erdorten eine andere Neigung besitzen werde, und meinte, der angenommene Zielpunkt werde sich als Schnittpunkt aller Neigungslinien berechnen lassen.

Auch Gilbert glaubte noch an eine regelmäßige Verteilung der Neigungen, und zwar sollten sie vom Äquator bis zu den geographischen Polen zuneh- 
men und sich daher für jede Breite vorausberechnen lassen, so daß ihre Werte umgekehrt zur Breitenbestimmung dienen würden.

Die ersten englischen Neigungsnadeln drehten sich samt ihrer waagrechten Achse zwischen einer Holz- und einer Glasscheibe, die mit einem Metallreifen ringsum verbunden und auf einen $\mathrm{Fu}$ gestellt waren, den man nach der magnetischen Abweichungsnadel richten mußte. Im 18. Jahrhundert wurden Neigungsgeräte vorgeschlagen, worin (wie beim Drähtchen im Wasser) dieselbe Nadel die Abweichung und Neigung zugleich angeben konnte. BuAcHE beschrieb 1732 eine solche Einrichtung. Auf einem senkrechten Stifte drehte sich das Hütchen hin und her. Dieses hatte zwei seitliche, aufgebogene Arme, die eine in Lagern bewegliche, waagrechte Achse zwischen sich faßten. Um diese Achse bewegte sich die Nadel auf und nieder. Ihre Fläche lag horizontal und war in der Mitte durchlocht, um nicht am Hütchen anzustoßen. Dabei waren die Reibungen zweier Drehungen zu überwinden. Coulomb verbesserte das Gerät 1777. Die Fläche seiner Nadel lag (wie schon bei Norman) vertikal. Sie war durch eine waagrechte Achse gesteckt, und diese drehte sich in Lagern in einem hufeisenförmigen Bügel, dessen Bogen sich oben befand, an einem Seidenfaden hing und sich daran ausrichtete.

Normans Entdeckung der Neigung leitete über zur Erkenntnis seines Landsmannes William Gilbert, daß die ganze Erdkugel ein großer Magnet sei. Gilberts Buch De Magnete von 1600 vereinigte alle bisherigen Kenntnisse über den Magneten und ging weit darüber hinaus. Der Verfasser war ein bekannter Arzt. In stiller nächtlicher Arbeit schuf er sein umwälzendes Werk. Er erweist sich darin als größter Erforscher des Magnetismus zwischen Peregrinus und Faraday, gleich bedeutend als Experimentator wie als Theoretiker. Er sonderte das Wirkliche vom Fabelhaften und deckte die Grundlagen der wahren Magnetlehre wieder auf, prägte Fachwörter, schrieb für künftige Forscher.

Gilbert setzte die Versuche mit dem kugelförmigen Magnetstein fort. Er hing ein Nädelchen an einen Faden und stellte die Magnetkugel darunter. Indem er sie in alle Lagen drehte, beobachtete er, wie das Nädelchen sich neigte. Über den Polen der Kugel stand es senkrecht; über dem magnetischen «Äquator» waagrecht und in den Zwischenlagen verschieden geneigt. Genau gleich schien das Verhalten der Neigungsnadel auf der Erdoberfläche zu sein. Gilbert meinte, am Äquator werde sich die Nadel waagrecht, an den Erdpolen senkrecht einstellen, und suchte die Neigungen in ein Gesetz zu fassen. 
Er betrachtete seine Magnetkugel als «Terrella», als kleines Erdmodell. Dieser Leitgedanke klingt durch sein ganzes Buch.

Da die Magnetkugel sich nicht drehte, setzte er zu Unrecht ihre magnetische Achse der geographischen Erdachse gleich. Die Lage der Erdachse und die Erddrehung betrachtete er als durch magnetische Himmelskräfte bedingt. Seine Ansicht, die Erde sei eine magnetische Kugel, traf zu; aber die Erscheinungen erwiesen sich später als verwickelter, indem die Erde gesonderte Magnetpole hatte. Gilbert lehnte diese ab; für ihn fielen sie mit den geographischen Polen zusammen.

Die Abweichung deutete er als bloße Störung der allgemeinen Richtung der Nadel nach den Erdpolen hin. Bei einem runden Magnetstein, dessen Oberfläche noch Löcher und Buckel aufwies, bemerkte er, daß die Nadel Abweichungen zeigte. Hielt man sie über eine Einbuchtung, so wandte sie sich dem nähern Beckenrande zu. In der Nähe eines Buckels wurde sie ebenfalls seitwärts angezogen, und zwar um so deutlicher, je näher die Unebenheit den Magnetpolen lag; denn jene Gebiete hatten größere magnetische Kräfte. Auch durch Gesteinseinsprenglinge wurden Ungleichheiten und Abweichungen bewirkt.

Gilbert übertrug alle diese Erfahrungen auf die Erde. Die Abweichungen gehorchten keiner festen Regel. Doch vom Atlantischen Ozean ließ sich sagen, daß die Nadeln sich jeweilen der nähern Festlandküste zuwandten. Und die Mißweisungen waren in den höhern Breiten größer. Gilbert folgerte bereits, daß die magnetische Kraft polwärts zunehmen müsse. Für ihn entsprach die Furche des Ozeans dem Loch in der Terrella, und die hervorragenden Festlandmassen entsprachen den Buckeln, die seitliche Zugkraft ausübten. Außerdem wirkten die verschiedenen Gesteine ungleich stark. Es lag kein Grund vor, eine zeitliche Änderung der Abweichungen anzunehmen.

Wie jeder Magnet seine Kraft auf Eisen überträgt, kann Eisen auch magnetisch werden dank der Magnetkraft der Erde. Daß Eisenkreuze auf Kirchen mit der Zeit magnetisch werden, vermochte Gilbert durch die Wirkung der Erdkraft zu erklären. Er sprach bereits von einem magnetischen Kraftfeld. Auch grenzte er die magnetische gegen die elektrische Anziehung ab. Eine neue Experimentierfreude hob an. Gilbert fand heraus, daß ein Magnetstein mehr Eisen zu heben imstande war, wenn man seine Pole mit Eisenkappen überzog oder armierte. GaliLeI, der diese Versuche wiederholte, erklärte die Erscheinung dadurch, daß die glattere Eisenkappe mehr wirksame Berührungspunkte bot als die stets etwas rauhere Oberfläche des Steines. 
Wie eine Offenbarung beflügelte Gilberts Buch den jungen Astronomen JoHANnes KePler, der vergeblich nach Gesetzen für die Abweichungswerte gesucht und auch einen Magnetpol berechnet hatte. Er hatte 1599 angenommen, daß ursprünglich die magnetische und geographische Erdachse zusammengefallen seien, da 3 sich aber dann die geographische um $6^{1 / 2} \mathrm{Grad}$ davon verschoben habe. Doch waren ihm seither Zweifel an der Regelmäßigkeit der Abweichungen und an der Wanderung der Polhöhe gekommen. Daher war er für Gilberts Werk vorbereitet. Er erhob die magnetische Anziehungskraft zum Sinnbilde der Schwerkraft. Ihm erschien der ganze Himmel mit magnetischen Kugeln bevölkert, die gegenseitig aufeinander einwirkten. Die Sonne sollte sich als große Magnetkugel inmitten der Planeten drehen und diese nach sich ziehen. Da die Planetenachsen ihre Richtung unverändert beibehalten und gegen die Ebene der Bahn um die Sonne geneigt sind, wenden die Planeten auf dem halben Wege ihres Umlaufes den einen, dann den andern Pol der Sonne zu und streben daher abwechselnd näher und ferner. Diese Vorstellungen leiteten Kepler zur Erkenntnis der elliptischen Bahnform. Erst Newton schied die Schwerkraft wieder vom Magnetismus, indem er zeigte, daß sie stets in gleichem Verhältnis zu den Massen steht, während die Magnetkraft den Massen ungleich zugeteilt ist.

Eine wichtige Berichtigung erfuhr Gilberts Lehre im 17. Jahrhundert durch die Erkenntnis der zeitlichen Veränderung der Abweichung. Der Mathematiker Henry Gellibrand schloß aus drei Messungen der Abweichung für London aus den Jahren 1580, 1622 und 1634, daß in dieser Zeitspanne von 54 Jahren die östliche Abweichung um über $7 \mathrm{Grad}$ zurückgegangen sei. Sie betrug bei der letzten Messung nur noch 4 Grad. In Nürnberg und anderswo wurde wenige Jahrzehnte später mit Staunen festgestellt, wie sich die Abweichung aus einer östlichen in eine westliche verwandelt hatte. Diese Veränderlichkeit erforderte fortgesetzte Nachprüfungen und führte zu planmäßigen Beobachtungen an verschiedenen Orten.

Um die folgende Jahrhundertwende erforschte Edmond Halley auf Seefahrten die Abweichungen im Atlantischen Ozean. Er traf vier Punkte ohne Abweichung, die sich in eine krumme Linie fassen ließen. Links davon lagen alle östlichen, rechts alle westlichen Abweichungen. Man konnte ziemlich gleichlaufende Linien ziehen, die die Punkte gleicher Abweichung miteinander verbanden. Auf Grund fremder Angaben zeichnete er die Linien auch für den Indischen Ozean ein. Halley legte seine Karte 1702 in die Hand der Seeleute als Hilfe für die Längenbestimmung. Er wußte, daß 
seine Abweichungslinien nur für beschränkte Zeit galten, aber gerade darum später geschichtliche Anhaltspunkte für die Richtung ihrer fortschreitenden Bewegung bieten mußten. Die Ursache für die zeitlichen Abweichungsveränderungen vermutete er im Innern der Erde. Dort mußte sich etwas verlagern. Er nahm einen Hohlraum und darin einen langsamer rotierenden magnetischen Kernkörper an.

Halleys graphische Darstellungsart wurde 1721 von WHISTon und 1786 von dem Schweden WILGKE auf die Neigungen übertragen, indem sie Punkte gleicher Neigung durch Linien verbanden.

Die Neigungen zeigten eine viel regelmäßigere Verteilung über die Erdoberfläche, und doch war sie verwickelter, als Gilbert gedacht hatte. Rings um die Erde verläuft allerdings eine «Linie ohne Neigung», wo die Magnetnadel waagrecht steht; aber dieser «magnetische Äquator» fällt nicht mit dem Erdäquator zusammen. Wilcke gab ihm in der Vorausberechnung die Gestalt eines Großkreises, der ungefähr 12 Grad gegen den Erdäquator geneigt war. In der Folgezeit trafen LAPEYrouse vor Brasilien und HumBOLDT in Peru je einen Punkt ohne Neigung an. Вгот legte 1804 durch diese beiden genau bestimmten Punkte einen größten Kreis um die Erde. Er suchte die Neigungen für verschiedene Orte in verschiedenem Abstand davon vorauszuberechnen und verglich sie mit gemessenen Werten. Seine Formel ließ notwendigerweise die örtlichen Störungen durch Gesteine außer acht. Senkrecht durch die Mitte der Ebene des angenommenen magnetischen Äquators zog er die magnetische Achse der Erde und sagte die Lage der Magnetpole voraus, das heißt jener Erdorte, wo die Neigungsnadel senkrecht stehen sollte.

Biots Anschauung vereinfachte die Wirklichkeit zu sehr, wie sich bald herausstellte. Da der magnetische Äquator im Atlantischen Ozean südlich des Erdäquators verlief und sich in Peru diesem näherte, hätte er im ganzen Stillen Ozean in der Nordhalbkugel verlaufen sollen. Dies traf nicht zu. Die Linie ohne Neigung war eine Kurve mit mehr Krümmungen. Zudem blieb sie nicht ruhig, sondern verschob sich im Laufe der Zeit. Von dem einen ihrer Schnittpunkte mit dem Äquator wurde an der Westküste Afrikas beobachtet, wie er allmählich in den Atlantischen Ozean hinaus fortschritt. In den Gebieten, denen er näherrückte, wurde die Neigung jährlich geringer, zum Beispiel in Paris.

Auch die Magnetpole lagen einander nicht genau gegenüber. JAmes Ross, der an der Fahrt seines Onkels zur Suche einer nordwestlichen Durchfahrt teilnahm, fand den nördlichen am 2. Juni 1831 am Kap Adelaide in Kanada 
(auf 70 Grad Breite und 96 Grad 46 Minuten westlicher Länge von Greenwich). Den südlichen erreichte erst Edgeworth David, ein Mitglied der Südpolarexpedition Shackletons, am 16. Januar 1909 im Innern des Südviktorialandes (in 74, Grad Breite und $145 \mathrm{Grad}$ östlicher Länge von Greenwich). Ungefähr dort hatte ihn schon James Ross vermutet, der 1841 auf der Suche danach jenes südlichste Land der Erde entdeckt hatte.

Damit war der alte Traum, daß man aus den Abweichungen oder Neigungen die Längen und Breiten einfach erschließen könne, zu Ende. Immerhin gestattet das Verfahren eine örtlich und zeitlich beschränkte Anwendung. Zu Halleys Zeit verliefen die Linien gleicher Abweichung bei Südafrika gleich wie die Meridiane, und zu Humboldts Zeit wechselten die Neigungen an den nebligen Küsten von Peru von Breite zu Breite. Daher erlaubten die Abweichungen bei Afrika die Schätzung der Länge und die Neigungen bei Peru die Schätzung der Breite.

Im 18. Jahrhundert begann man außer den Richtungswinkeln der Nadeln auch die Kräfte zu messen, mit denen die Erde diese Richtungsweisung erzwang. Schon Gilbert hatte geahnt, daß diese Kräfte gegen die höhern Breiten hin zunehmen sollten, daß also die Erde nicht überall gleich kräftig auf die Magnetnadeln wirke. Die beiden Pole der Magnetsteine zeigten sich in London auch nie gleich stark; der nach Süden gerichtete war hier immer der stärkere.

Um aber die Kräfte messend zu vergleichen, mit denen eine Nadel zu einer bestimmten Zeit an verschiedenen Erdorten gelenkt wurde, besaß man noch kein Verfahren. Das 17. Jahrhundert nun entwickelte die Pendeluhr, die von der Schwerkraft angetrieben wird. Hierbei fand sich, daß das Pendel am Äquator oder auf hohen Bergen langsamer schwang, da offenbar die anziehende Kraft der Erdmasse mit der Entfernung vom Erdmittelpunkt abnahm. Die in gleichen Zeitspannen ausgeführte Anzahl der Schwingungen eines bestimmten um die Ruhelage pendelnden Körpers stellte also allgemein ein Mittel dar, anziehende Kräfte zahlenmäßig auszudrücken. Eine Abweichungs- oder Neigungsnadel, die man an verschiedene Erdorte hintrug, um sie in Schwingungen um ihre Ruhelage zu versetzen, mußte an Erdstellen schwächerer Intensität langsamere Schwingungen zeigen als an Orten stärkerer erdmagnetischer Kraft. Dabei wurden die Schwingungen einer Neigungsnadel, da diese genau in der Richtung der magnetischen Zugkraft einspielen konnte, von der örtlichen Gesamtkraft des Erdmagnetismus hervorgerufen und ergaben unmittelbar das Maß dafür. Die horizontale Nadel stand dagegen nur unter dem Einfluß der waagrechten Kom- 
ponente. Doch ließ sich aus ihren Schwingungen, wenn man zugleich den Wert der Neigung maß, die Totalkraft der Resultante berechnen, indem man die Horizontalkraft durch den Kosinus des Neigungswinkels dividierte.

SAussure glaubte 1788, mit einem magnetischen Pendel auf dem Col du Géant eine Abnahme der magnetischen Erdkraft in der Höhe festzustellen. Spätere Gebirgsreisende fanden jedoch zuweilen auch eine Zunahme. Diese konnte durch die Beschaffenheit der Gesteine bedingt sein, da ja der Magnetismus auf die Stoffe der Erdrinde ungleich verteilt ist. Um diesen Einfluß auszuschalten, prüfte man die Schwingungen der Magnetnadel auf Ballonfahrten. Man erhielt keine Verlangsamung, beachtete aber nicht, daß infolge der Kälte in den höheren Luftschichten die Nadel sich verkürzte und daher, wenn sie noch unter gleichen Kräften gestanden hätte, schneller hätte schwingen müssen. FARAdAY wies darauf hin, daß in einem starken Magnetfeld sämtliche Stoffe sich richten, und zwar die Gase ostwestlich, dagegen der Sauerstoff gleich wie das Eisen nordsüdlich. Da nun die Sauerstoff hülle der Erde in größerer Höhe weniger dicht ist, muß dieser Umstand mithelfen, die atmosphärische Richtkraft in der Höhe zu schwächen. Freilich wird andererseits durch die Kälte die Richtkraft des Sauerstoffs erhöht. Dies muß sich besonders nachts auswirken, auf der augenblicklich von der Sonne abgewandten Erdhälfte.

Schon vor Saussures Versuch wollte BoRDA mit einer Neigungsnadel die Erdkraft an weit auseinanderliegenden Erdorten vergleichen. Doch sah er bald, daß er zuerst eine Neigungsnadel mit verminderter Reibung anfertigen lassen mußte, die man zugleich genauer aufstellen konnte. Mit diesen verbesserten Nadeln rüstete er Reisende aus, darunter Alexander von НuмвоцDт, der diese «Boussole d'inclinaison» mit nach Südamerika nahm. Unterwegs zeigte es sich, daß die Nadel im allgemeinen langsamer schwang, je weniger sie sich neigte. Am langsamsten schwang sie an dem von Humboldt erreichten Punkte des magnetischen Äquators. Dies verleitete ihn zu der Verallgemeinerung, daß die Erdkraft von jener Kurve aus bis zu den Magnetpolen ununterbrochen wachse und daß Unregelmäßigkeiten nur ganz örtlichen Einflüssen zuzuschreiben seien. Humboldt teilte den von ihm bereisten Teil Südamerikas in Zonen ein, unter denen die mittlere magnetische Kraft mit zunehmender Entfernung vom magnetischen Äquator abnahm. Die Schwingungszahl, die er am Punkte der Kurve ohne Neigung in Peru erhalten hatte, setzte er als Krafteinheit fest, und er drückte die Schwingungszahlen der übrigen Orte mit Dezimalbrüchen in bezug auf diese Einheit aus. In Paris betrug demnach die Erdkraft 1,3482. 
Im Atlantischen Ozean klärte Edward Sabine die magnetischen Erdkraftverhältnisse auf. Es ergab sich, daß die Linien gleicher Erdkraft nicht mit den Linien gleieher Neigung übereinstimmten. Die verschiedenen Punkte ohne Neigung zeigten ungleiche Kräfte, und die Punkte schwächster Intensität jedes Meridians fielen nicht mit den Punkten ohne Neigung zusammen, sondern bildeten, wenn man sie von Meridian zu Meridian miteinander verband, eine eigene Wellenlinie, den «dynamischen Äquator». Die ersten Karten mit Isodynamen oder Linien gleicher magnetischer Erdkraft lieferte der Norweger Hansteen. Auf jeder Hemisphäre scharten sich die geschlossenen Linien immer größerer Erdkraft zuletzt um je zwei gesonderte Brennpunkte größter Kräfte. Diese vier Zentren hatten untereinander verschiedene Stärke.

An Stelle von Humboldts Einheit, die sich als willkürlich erwies, schlug Gauss eine absolute magnetische Krafteinheit vor. Sie ist dadurch bestimmt, daß sie einer gleichen Kraft im Abstande 1 die Beschleunigung 1 erteilt. Auch in dieser Einheit läßt sich die Kraft einer Erdstelle nur mit vorübergehender Gültigkeit angeben, da sie sich ebenso wie die Abweichung und Neigung dauernd verändert.

Die Abweichung, Neigung und Erdkraft wandeln sich nicht nur jährlich, sondern auch stündlich. Für die Abweichung und Neigung erkannte dies der Londoner Uhrmacher George Graham 1722/23, für die Erdkraft durch Zählung der Nadelschwingungen Christopher Hansteen 1821.

In Europa wanderte die nördliche Spitze der Abweichungsnadel vormittags nach Westen, nachmittags nach Osten. Mittags gegen $2 \mathrm{Uhr}$ zeigte sie in Berlin die größte westliche Abweichung. Dann näherte sie sich der Nordrichtung bis morgens $8 \mathrm{Uhr}$. Unterwegs führte sie, wie НuмвоLDT entdeckte, nach Mitternacht noch eine Zwischenschwankung aus, indem sie zwei Stunden rückwärts ging, bevor sie ihren östlichen Weg fortsetzte. Durch den Vormittag eilte sie dann wieder in die größte Abweichung zurück. An verschiedenen Orten der Südhalbkugel lief die Nadel vor- und nachmittags gerade in umgekehrter Richtung als in Europa. Man vermutete daher, es gebe unter den Tropen rings um die Erde eine Kette von Orten ohne stündliche Schwankung. Doch fand sich statt dessen nur eine Reihe von Stellen, wo die Schwankungen bald wie im Norden, bald wie im Süden geschahen. Auf Sankt Helena verhielt sich die Nadel von Mai bis September deutlich wie in Europa, von Oktober bis Februar wie in Tasmanien.

Außer den regelmäßigen Schwankungen traten unregelmäßige auf, heftige "magnetische Ungewitter», meist nach Mitternacht, und zwar auf 
der Nordhalbkugel mehr im April bis September, im Süden dagegen mehr vom Oktober bis März, also überall mehr in der wärmern Jahreszeit, wo der entsprechende Erdpol sich der Sonne zuwendet.

Bei starken magnetischen Gewittern beobachtete man zugleich im Gebiet der Magnetpole der Erde das Auftreten von Nord- und Südlichtern. Es handelt sich dabei um ein Ausströmen der aufgehäuften Erdelektrizität in den Luftraum, wobei der Planet zum leuchtenden Körper wird.

Die täglichen Schwankungsweiten der Nadel und die Anzahl der Gewitter blieben sich im Jahresmittel nicht gleich. Sie nahmen ungefähr fünf Jahre zu und dann wieder ungefähr fünf Jahre $a b$, in einer elfjährigen Periode. Genau dieselbe Periode offenbarte sich in der zunehmenden und abnehmenden jährlichen Häufigkeit der Sonnenflecken.

Sabine entdeckte, daß auf der ganzen Erde die magnetische Kraft vom Oktober bis Februar am stärksten ist. Die Erde kommt um diese Zeit in ihrer Ellipsenbahn der Sonne am nächsten. Deren Nähe steigert die Kräfte. Die Sonne scheint um sich ein Magnetfeld zu verbreiten, wie Kepler geahnt hat. Zugleich wächst mit der Nähe der Wärmequelle auch die elektrische Ladung. Denn infolge der täglichen Drehung der Erde erhält ihre Oberfläche eine wechselnde Erwärmung. Diese erzeugt elektrische Ströme, und zwar um so mehr, je ausgesprochener Wärme und Kälte sich folgen.

So wird die Erde von seltsamen Kräften umspielt. Licht und Wärme, Elektrizität und Magnetismus verwandeln sich geheimnisvoll ineinander, und ein unendliches Staunen erfüllt den Betrachter*.

* Der Aufsatz von Heinz BaLmer stellt eine gedrängte Übersicht seines großen, in der Serie unserer Gesellschaft als Band XX erscheinenden Werkes Beiträge zur Geschichte der Erkenntnis des Erdmagnetismus dar, welches im Herbst dieses Jahres im Verlag H. R. Sauerländer \& Co., Aarau, herauskommen wird (siehe 4. Umschlagseite).

Der Redaktor 